\title{
Hybrid Hydrogels with Extremely High Stiffness and Toughness
}

\section{Citation}

Li, Jianyu, Widusha R. K. Illeperuma, Zhigang Suo, and Joost J. Vlassak. 2014. “Hybrid Hydrogels with Extremely High Stiffness and Toughness." ACS Macro Lett. (May 19): 520-523. doi:10.1021/ mz5002355.

\section{Published Version}

doi:10.1021/mz5002355

\section{Permanent link}

http://nrs.harvard.edu/urn-3:HUL.InstRepos:13910151

\section{Terms of Use}

This article was downloaded from Harvard University's DASH repository, and is made available under the terms and conditions applicable to Open Access Policy Articles, as set forth at http:// nrs.harvard.edu/urn-3:HUL.InstRepos:dash.current.terms-of-use\#OAP

\section{Share Your Story}

The Harvard community has made this article openly available.

Please share how this access benefits you. Submit a story.

Accessibility 


\title{
Hybrid hydrogels with extremely high stiffness and toughness
}

\author{
Jianyu Li, Widusha R.K. Illeperuma, Zhigang Suo* and Joost J. Vlassak* \\ School of Engineering and Applied Sciences, Harvard University, Cambridge, MA 02138, USA \\ KEYWORDS: hydrogels, fracture mechanics, toughness, stiffness, mechanical properties
}

\begin{abstract}
The development of hydrogels for cartilage replacement and soft robotics has highlighted a challenge: load-bearing hydrogels need to be both stiff and tough. Several approaches have been reported to improve the toughness of hydrogels, but simultaneously achieving high stiffness and toughness remains difficult. Here we report that alginate-polyacrylamide hydrogels can simultaneously achieve high stiffness and toughness. We combine short- and long-chain alginates to reduce the viscosity of pre-gel solutions, and synthesize homogeneous hydrogels of high ionic crosslink density. The resulting hydrogels can have elastic moduli of $\sim 1 \mathrm{MPa}$ and fracture energies of $\sim 4 \mathrm{~kJ} \mathrm{~m}^{-2}$. Furthermore, this approach breaks the inverse relation between stiffness and toughness: while maintaining constant elastic moduli, these hydrogels can achieve fracture energies up to $\sim 16 \mathrm{~kJ} \mathrm{~m}^{-2}$. These stiff and tough hydrogels hold promises for the further development as load-bearing materials.
\end{abstract}


Hydrogels are used as scaffolds in tissue engineering, ${ }^{1}$ carriers for drug delivery, ${ }^{2}$ valves in microfluidics, ${ }^{3}$ and superabsorbent polymers in disposable diapers. ${ }^{4}$ Many other applications require hydrogels of exceptional mechanical properties. Examples include biomedical applications such as materials for cartilage replacement, ${ }^{5}$ engineering applications such as swellable packers for oil and gas recovery, ${ }^{6}$ and artificial muscles and artificial nerves in the nascent field of soft machines. ${ }^{7-9}$ These load-bearing applications of hydrogels are often limited by low stiffness and toughness. ${ }^{10}$ Hydrogels for cartilage replacement, for instance, require high stiffness and toughness to retain shape and to resist fracture, respectively. ${ }^{5}$ Several approaches have been reported to improve the toughness of hydrogels, ${ }^{11-14}$ but simultaneously achieving high stiffness and toughness remains a challenge. Stiffness and toughness of polymer networks are often inversely related. According to the Lake-Thomas model, for example, as the crosslink density decreases, toughness increases, but stiffness decreases. ${ }^{15}$ Most hydrogels are either stiff and brittle with low fracture energies on the order of $10 \mathrm{~J} \mathrm{~m}^{-2}$, or tough and compliant with low elastic moduli on the order of $10 \mathrm{kPa}$. To place these values in context, note that cartilage has elastic moduli on the order of $1,000 \mathrm{kPa}$ and fracture energies on the order of $1,000 \mathrm{~J} \mathrm{~m}^{-2} \cdot{ }^{16,17}$

It has been discovered that hydrogels can achieve high toughness by using double networks. ${ }^{11,18}$ A recent work has shown that alginate-polyacrylamide hydrogels can achieve fracture energy of $9,000 \mathrm{~J} \mathrm{~m}^{-2} \cdot{ }^{13}$ Tests of biocompatibility have encouraged further exploration of the potential of alginate-polyacrylamide hydrogels as biomaterials. ${ }^{19}$ For example, these hydrogels have been infused into a scaffold of woven fibers to mimic cartilage. ${ }^{20}$ Although the alginate-polyacrylamide hydrogels have achieved exceptionally high toughness, their stiffness is modest for three reasons. First, the concentration of alginate in the hydrogel is low; any attempt to significantly raise the concentration of alginate is frustrated by the high viscosity, making it difficult to mix the ingredients to form a homogeneous hydrogel. Second, the crosslink density 
of alginate is low because the crosslinker, calcium sulfate, has low solubility. Third, as mentioned above, stiffness and toughness are often inversely related. As the stiffness increases, the toughness decreases significantly.

Here we report that alginate-polyacrylamide hydrogels can simultaneously achieve high stiffness and toughness. We increase the concentration of alginate while maintaining relatively low viscosity by using both short- and long-chain alginates. We increase the crosslink density of alginate while maintaining homogeneous distribution of alginate by using a combination of calcium sulfate and calcium chloride as crosslinkers. This approach breaks the inverse relation between stiffness and toughness. The resulting hydrogels achieve stiffness and toughness significantly beyond those of existing hydrogels (Figure 1).



Figure 1. Fracture energies and elastic moduli of various materials. The hybrid gels in the current work are compared with other soft materials, including hybrid gels with long-chain alginate only, ${ }^{13}$ double network hydrogels, ${ }^{18,35}$ polyvinyl alcohol hydrogels (PVA) ${ }^{36}$ cartilage, ${ }^{16,17}$ natural 
elastomers, ${ }^{17,37}$ skin, ${ }^{17,38}$ cork $^{17}$ along with data for polyacrylamide hydrogels (PAAm) and alginate hydrogels. $^{13}$

An alginate-polyacrylamide hydrogel consists of two interpenetrating polymer networks. Polyacrylamide forms a covalently crosslinked network (Figure 2a). Alginate is a linear block copolymer of 1,4-linked $\beta$-D-mannuronic acid $(\mathrm{M})$ and $\alpha$-L-guluronic acid $(\mathrm{G})$ residues. $^{21}$ This block copolymer forms crosslinks via ionic interactions between the $\mathrm{G}$ residues on the chains and chelating ions such as $\mathrm{Ca}^{2+}$ (Figure $\left.2 \mathrm{~b}\right){ }^{22,23}$ The high toughness results from the synergy of two mechanisms: crack bridging by the covalently crosslinked polyacrylamide network and energy dissipation by unzipping ionic crosslinks in the alginate network over a large region of the hydrogel (Figure 2c). ${ }^{13}$
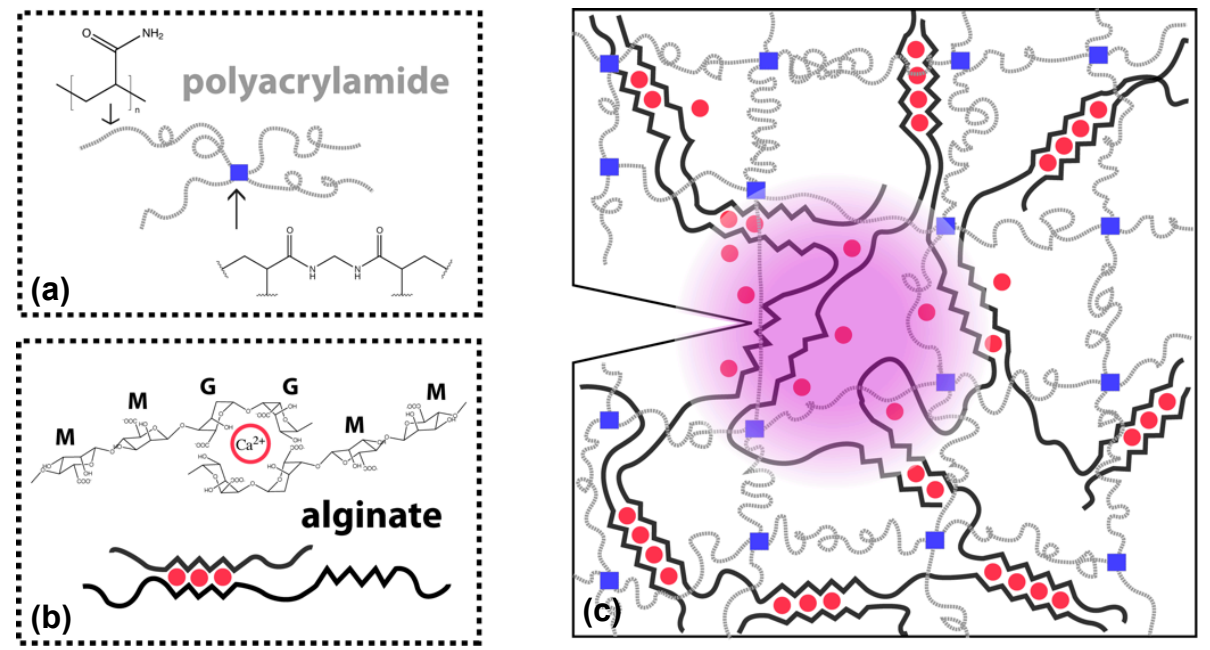

Figure 2. Structure and toughening mechanism of hybrid hydrogels. a, Polyacrylamide (PAAm; grey dashed lines) forms covalent crosslinks through N,N`-methylenebisacrylamide (MBAA; blue squares). b, Alginate chains (black lines) consisting of $G$ blocks form ionic crosslinks through $\mathrm{Ca}^{2+}$ (red circles). c, A large area of plastic zone (pink region) presents ahead of the notch where ionic crosslinks unzip to dissipate energy. 
The viscosity of an aqueous solution of alginate depends on both the concentration and chain length of the alginate. ${ }^{24-26}$ Alginate is a natural product available only with specific chain lengths. Modification of the chain length requires special treatments such as $\gamma$-ray irradiation, which may also introduce changes in the distribution of the uronate residues. ${ }^{27}$ We used two types of biologically derived alginates that differ in the chain length, but not in the distribution of the uronate residues: LF20/40, a long-chain alginate with a molecular weight of $200 \mathrm{~kg} \mathrm{~mol}^{-1}$; and LFR5/60, a short-chain alginate with a molecular weight of $30 \mathrm{~kg} \mathrm{~mol}^{-1} \cdot{ }^{28,29}$ Both alginates are commercially available (FMC BioPolymer). In an aqueous solution containing both alginates, the viscosity reduces as the fraction of the short-chain alginate increases (Supporting Information, Figure S1). The solution of the short-chain alginate has a low viscosity even at a high concentration of alginate (Supporting Information, Figure S2).

We increase the ionic crosslink density by using a combination of calcium sulfate and calcium chloride. The crosslinker used in our previous work, $\mathrm{CaSO}_{4} \cdot 2 \mathrm{H}_{2} \mathrm{O}$, has a low solubility (2.4 $\mathrm{g} \mathrm{L}^{-1}$ at $\left.20^{\circ} \mathrm{C}\right) .^{13}$ The solubility of $\mathrm{CaCl}_{2}$ is high, but the direct use of $\mathrm{CaCl}_{2}$ as crosslinker causes inhomogeneous distribution of alginate in pure alginate hydrogels. ${ }^{26,30}$ Here we show that $\mathrm{CaCl}_{2}$ can be used to synthesize alginate-polyacrylamide hydrogels with homogeneous distribution of alginate. Our new protocol of synthesis is as follows. A homogeneous alginatepolyacrylamide hydrogel was first formed with $\mathrm{CaSO}_{4} \cdot 2 \mathrm{H}_{2} \mathrm{O}$; the crosslink density of G blocks was low, but alginate chains were immobilized in the network. Subsequently, the hydrogel was immersed in a large volume of a $1.0 \mathrm{M} \mathrm{CaCl}_{2}$ solution to achieve full crosslinking of $\mathrm{G}$ blocks, while retaining the homogeneous distribution of alginate in the hydrogel. This observation is consistent with a recent report. ${ }^{31}$

We demonstrate that the concentration of alginate strongly affects the properties of alginate-polyacrylamide hydrogels. In a series of experiments, we fixed the ratio of 
polyacrylamide to water at $16.8 \mathrm{wt} \%$ and fixed the ratio of short-chain to long-chain alginates at 4:1, but varied the concentration of alginate. The G blocks on the alginate chains were fully crosslinked. We then performed a series of tensile tests on both unnotched and notched samples. For the tensile tests of unnotched samples, as the concentration of alginate increases, the strength increases, but the rupture stretch decreases (Figure 3a). The nominal stress is the loading force divided by the cross-sectional area of the undeformed sample. The stretch is the current length divided by the initial length of undeformed sample. The elastic modulus is the slope of the initial portion of the stress-stretch curves. As the alginate content increases, the elastic modulus of the hydrogel increases (Figure 3b). The fracture energy of the hydrogel was measured by performing tensile tests on notched samples using a geometry known as the pure shear test. ${ }^{13,32}$ The fracture energy of the hydrogel decreases with increasing alginate content (Figure 3c). Despite this reduction, the hydrogel with the maximum alginate content maintains a large fracture energy of $4 \mathrm{~kJ} \mathrm{~m}^{-2}$, while exhibiting a large elastic modulus of $1,000 \mathrm{kPa}$.
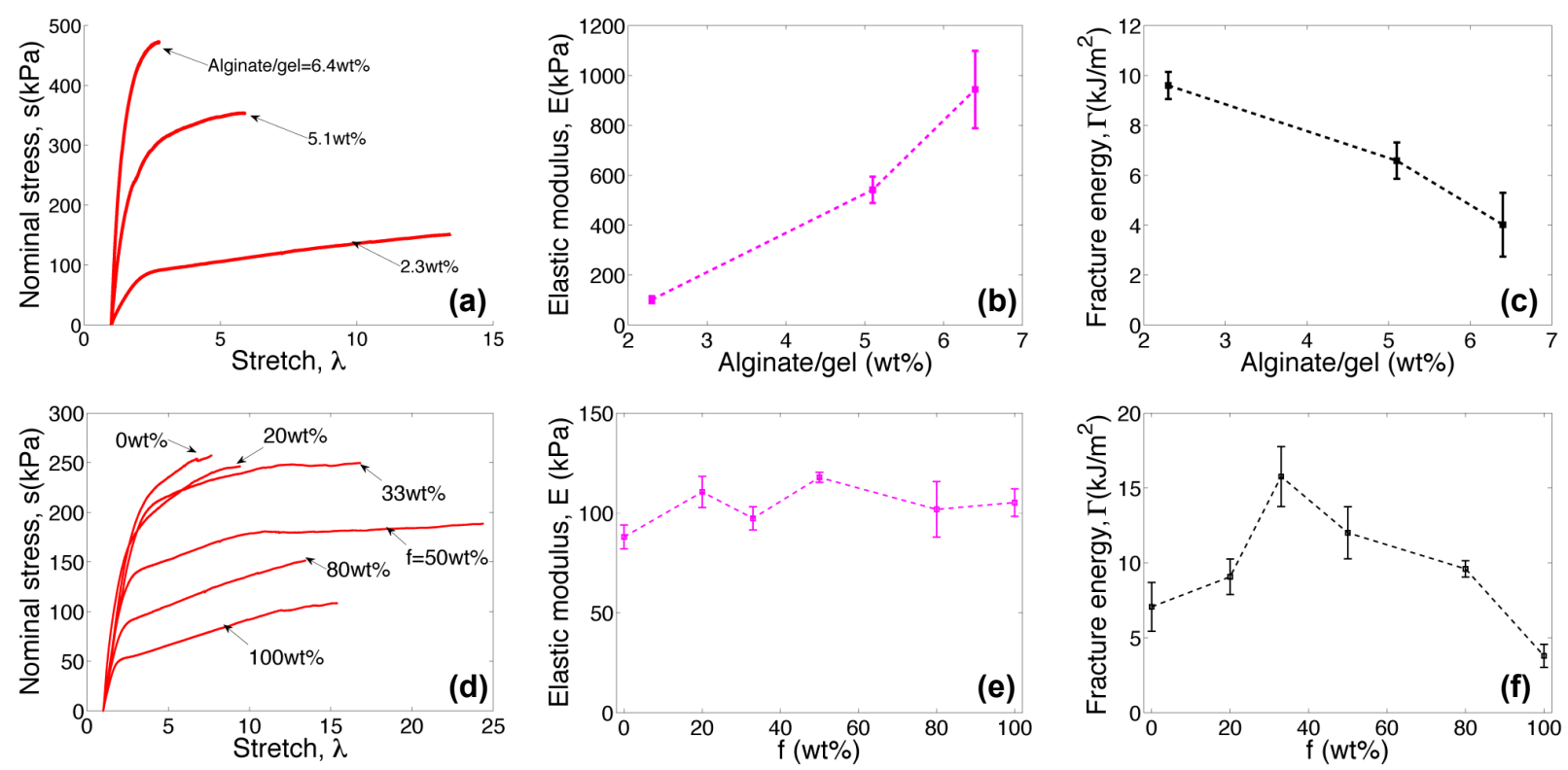

Figure 3. Properties of hybrid hydrogels. a, Stress-stretch curves of gels of various concentrations of alginate. Each test was performed by pulling an unnotched sample to rupture. $b$, 
Elastic modulus as a function of the concentration of alginate in the gel. c, Fracture energy varies with the concentration of alginate. d, Stress-stretch curves of gels of various fractions of shortchain alginate to the total alginate. e,f, Elastic modulus (e) and fracture energy (f) vary with the fraction of short-chain alginate. Error bars show standard deviation; sample size $n=4$.

We interpret this inverse relation between stiffness and toughness as follows. Increasing the alginate content not only raises the stiffness of the hydrogel, but also its strength (Figure 3a). The high strength, in turn, lowers the toughness of the hydrogel because the size of the process zone, i.e., the region near the crack tip where dissipative processes take place, scales inversely with the strength of a material. The trade-off between strength and toughness is well recognized in materials science. ${ }^{33,34}$

Our new approach breaks this inverse relation between stiffness and toughness. In pure alginate hydrogels, the chain length affects strength, but not stiffness (Supporting Information, Figure S3). The same phenomenon occurs in alginate-polyacrylamide hydrogels. We varied the weight percentage of short-chain alginate in the gels, $f$, while fixing the total weight percentage of alginate at $2.3 \mathrm{wt} \%$ and the ratio of $\mathrm{CaSO}_{4}$ to alginate at $20 \mathrm{wt} \%$. The strength of the hydrogel decreases as the fraction of short-chain alginate increases (Figure 3d), while the elastic modulus is independent of the fraction of short-chain alginate (Figure 3e). The fracture energy reaches a maximum value of $16 \mathrm{~kJ} \mathrm{~m}^{-2}$ at an intermediate proportion of short- and long-chain alginates (Figure 3f). The maximum value of the fracture energy is a twofold improvement over the highest value reported before for alginate-polyacrylamide hydrogels with long-chain alginate only. ${ }^{13}$ It is perhaps the first time that a hydrogel containing around $90 \%$ water rivals natural elastomers in toughness (Figure 1). Furthermore, we can independently optimize the stiffness and 
toughness of a hydrogel by varying the molecular weight and crosslink density of the alginate (Supporting Information, Figure S4).

We interpret the observed relation between the fracture energy and the fraction of shortchain alginate as follows. In the alginate-polyacrylamide hydrogel containing only long-chain alginate, the strength is so high that only a small region around the root of the notch is stressed enough to unzip ionic crosslinks, leading to a low fracture energy (Supporting Information, Figure S5a). As the fraction of short-chain alginate increases, the strength decreases and the size of the region in which ionic crosslinks unzip grows, leading to an increase in fracture energy (Supporting Information, Figure S5b). When the content of the short-chain alginate is too large, the strength of the alginate network is too low to effectively contribute to energy dissipation. Thus the fracture energy goes through a maximum at an intermediate fraction of short-chain alginate.

In summary, this work demonstrates a significant improvement of stiffness and toughness of alginate-polyacrylamide hydrogels. By using a combination of short- and long-chain alginates, calcium sulfate and calcium chloride as crosslinkers, we can increase the concentration of alginate and ionic crosslink density, and optimize the stiffness and toughness of hydrogels independently. These hybrid hydrogels are accessible to a very large range of stiffness and toughness combinations, extending significantly beyond traditional benchmarks. The combination of high stiffness, high toughness, biocompatibility and facile synthesis makes these hydrogels ideal candidates for further development as load-bearing materials.

\section{EXPERIMENTAL SECTION}

Hydrogel synthesis. Alginate-polyacrylamide hydrogels were synthesized using the following protocol. Sodium alginate (LFR5/60 and LF20/40) and acrylamide (AAm) were dissolved in 
distilled water. The weight of AAm was fixed at 0.168 times that of water. The mixture was stirred for 2 days at room temperature until the solution became homogeneous. The mixture was subsequently mixed with N,N`-methylenebisacrylamide (MBAA), tetramethyl-ethylenediamine (TEMED), $\mathrm{CaSO}_{4} \cdot 2 \mathrm{H}_{2} \mathrm{O}$ and ammonium persulfate (APS) in this sequence. The weights of MBAA, TEMED and APS were fixed at $0.0006,0.0055$ and 0.006 times that of AAm. The weight of alginate and $\mathrm{CaSO}_{4}$ was varied as noted earlier. The mixture was then placed in a glass mold (75 x $45 \times 3 \mathrm{~mm}^{3}$ ) and cured by UV (OAI LS 30 UV flood exposure system, 350W, wavelength $365 \mathrm{~nm}$ ) for $200 \mathrm{~s}$. The product was kept at room temperature overnight to ensure complete reaction. A combination of $\mathrm{CaSO}_{4}$ and $\mathrm{CaCl}_{2}$ was used as crosslinkers; the concentration of $\mathrm{CaSO}_{4} \cdot 2 \mathrm{H}_{2} \mathrm{O}$ was fixed at $19.4 \mathrm{mM}$ during gelation, and then the samples were submersed in a large volume of $1.0 \mathrm{M} \mathrm{CaCl}_{2}$ solution for at least 3 days.

Mechanical testing. A rectangular strip of the hybrid hydrogel $\left(75 \times 45 \times 3 \mathrm{~mm}^{3}\right)$ was glued to two rigid acrylate clamps $\left(75 \times 20 \times 1.5 \mathrm{~mm}^{3}\right)$. For notched samples, an edge crack of length $35 \mathrm{~mm}$ was cut using a razor blade in the middle of the gauge section of the sample. An Instron machine (model 3342 with load cell of maximum 1000N) was used for tensile tests. The stretch rate was $2 \mathrm{~min}^{-1}$. The signals of force and extension were recorded by the Instron machine throughout the test.

\section{ASSOCIATED CONTENT}

Supporting Information Available: Viscosity of alginate solutions, compression tests of alginate hydrogels, effects of ionic crosslink density, effects of alginate chain length. This material is available free of charge via the Internet at http://pubs.acs.org. 


\section{AUTHOR INFORMATION}

\section{Corresponding Author}

*suo@seas.harvard.edu.

*vlassak@seas.harvard.edu.

\section{Author Contributions}

The manuscript was written through contributions of all authors.

\section{ACKNOWLEDGMENT}

The work was supported by the MRSEC (DMR-0820484) and the Kavli Institute for Bionano

Science and Technology at Harvard University. It was performed in part at the Center for Nanoscale Systems at Harvard University. The authors thank David A. Weitz for providing the rheometer and David J. Mooney for providing the Instron machine.

\section{REFERENCES}

1 Park, H.; Kang, S. W.; Kim, B. S.; Mooney, D. J.; Lee, K. Y. Macromol. Biosci., 2009, 9, 895-901.

2 Langer, R. Nature, 1998, 392, 5-10.

3 Beebe, D. J.; Moore, J. S.; Bauer, J. M.; Yu, Q.; Liu, R. H.; Devadoss, C.; Jo, B. H. Nature, 2000, 404, 588-590.

4 Dubrovskii, S. A.; Afanaseva, M. V.; Lagutina, M. A.; Kazanskii, K. S. Polym. Bull., 1990, 24, 107-113.

5 Huey, D. J.; Hu, J. C.; Athanasiou, K. A. Science, 2012, 338, 917-921.

6 Kleverlaan, M.; van Noort, R. H.; Jones, I. in SPE/IADC Drilling Conference, Society of Petroleum Engineers, 2005. 
7 Lee, H.; Xia, C.; Fang, N. X. Soft Matter, 2010, 6, 4342-4345.

8 Shepherd, R. F.; Ilievski, F.; Choi, W.; Morin, S. A.; Stokes, A. A.; Mazzeo, A. D.; Chen, X.; Wang, M.; Whitesides, G. M. PNAS, 2011, 108, 20400-20403.

9 Keplinger, C.; Sun, J. Y.; Foo, C. C.; Rothemund, P.; Whitesides, G. M.; Suo, Z. G. Science, 2013, 341, 984-987.

10 Calvert, P. Adv. Mater., 2009, 21, 743-756.

11 Gong, J. P.; Katsuyama, Y.; Kurokawa, T.; Osada, Y. Adv. Mater., 2003, 15, 1155-1158.

12 Henderson, K. J.; Zhou, T. C.; Otim, K. J.; Shull, K. R. Macromolecules, 2010, 43, 61936201.

13 Sun, J. Y.; Zhao, X. H.; Illeperuma, W. R. K.; Chaudhuri, O.; Oh, K. H.; Mooney, D. J.; Vlassak, J. J.; Suo, Z. G. Nature, 2012, 489, 133-136.

14 Zhao, X. H. Soft Matter, 2014, 10, 672-687.

15 Lake, G. J.; Thomas, A. G. Proc. R. Soc. London, Ser. A, 1967, 300, 108-119.

16 ChinPurcell, M. V.; Lewis, J. L. J. Biomech. Eng., 1996, 118, 545-556.

17 Wegst, U. G. K.; Ashby, M. F. Philos. Mag., 2004, 84, 2167-2181.

18 Gong, J. P. Soft Matter, 2010, 6, 2583-2590.

19 Darnell, M. C.; Sun, J. Y.; Mehta, M.; Johnson, C.; Arany, P. R.; Suo, Z. G.; Mooney, D. J. Biomaterials, 2013, 34, 8042-8048.

20 Liao, I.; Moutos, F. T.; Estes, B. T.; Zhao, X. H.; Guilak, F. Adv. Funct. Mater., 2013, 23, 5833-5839.

21 Rowley, J. A.; Madlambayan, G.; Mooney, D. J. Biomaterials, 1999, 20, 45-53.

22 Webber, R. E.; Shull, K. R. Macromolecules, 2004, 37, 6153-6160.

23 Zhao, X. H.; Huebsch, N.; Mooney, D. J.; Suo, Z. G. J. Appl. Phys., 2010, 107, 063509. 
24 Draget, K. I.; Simensen, M. K.; Onsoyen, E.; Smidsrod, O. Hydrobiologia, 1993, 261, 563-569.

25 Draget, K. I.; Braek, G. S.; Smidsrod, O. Carbohydr. Polym., 1994, 25, 31-38.

26 Kuo, C. K.; Ma, P. X. Biomaterials, 2001, 22, 511-521.

27 Kong, H. J.; Kaigler, D.; Kim, K.; Mooney, D. J. Biomacromolecules, 2004, 5, 1720-1727.

28 Manojlovic, V.; Djonlagic, J.; Obradovic, B.; Nedovic, V.; Bugarski, B. Int. J. Nanomed., 2006, $1,163-171$.

29 Johnson, F. A.; Craig, D. Q. M.; Mercer, A. D.; Chauhan, S. Int. J. Pharm., 1997, 159, $35-42$.

30 Skjåk-Bræk, G.; Grasdalen, H.; Smidsrød, O. Carbohydr. polym., 1989, 10, 31-54.

31 Yang, C. H.; Wang, M. X.; Haider, H.; Yang, J. H.; Sun, J. Y.; Chen, Y. M.; Zhou, J.; Suo, Z. ACS appl. mater. Inter., 2013, 5, 10418-10422.

32 Rivlin, R. S.; Thomas, A. G. J. Polym. Sci., 1953, 10, 291-318.

33 Tvergaard, V.; Hutchinson, J. W. J. Mech. Phys. Solids, 1992, 40, 1377-1397.

34 Ritchie, R. O. Nat. Mater., 2011, 10, 817-822.

35 Nakajima, T.; Furukawa, H.; Tanaka, Y.; Kurokawa, T.; Osada, Y.; Gong, J. P. Macromolecules, 2009, 42, 2184-2189.

36 Zhang, L.; Zhao, J.; Zhu, J. T.; He, C. C.; Wang, H. L. Soft Matter, 2012, 8, 10439-10447.

37 Hamed, G. R.; Han, K. T. Rubber Chem. Technol., 1990, 63, 806-824.

38 Koutroupi, K. S.; Barbenel, J. C. J. Biomech., 1990, 23, 281-287. 


\section{for Table of Contents use only}

Hybrid hydrogels with extremely high stiffness and toughness

Jianyu Li, Widusha R.K. Illeperuma, Zhigang Suo* and Joost J. Vlassak*

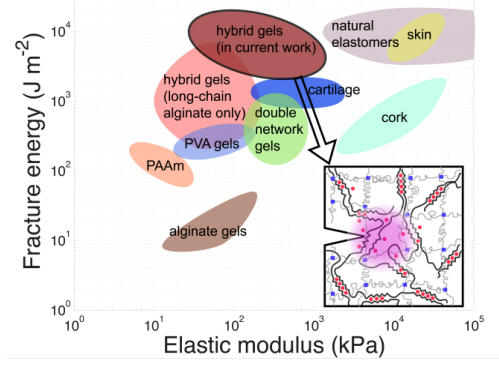

